\title{
NOTIONS OF CONTROLLABILITY FOR QUANTUM MECHANICAL SYSTEMS.
}

\author{
Francesca Albertini \\ Dipartimento di Matematica Pura ed Applicata, \\ Universita' di Padova, \\ via Belzoni 7 , \\ 35100 Padova, Italy. \\ Tel. (+39) 0498275966 \\ email: albertin@math.unipd.it \\ Domenico D'Alessandro \\ Department of Mathematics \\ Iowa State University \\ Ames, IA 50011, USA \\ Tel. (+1) 5152948130 \\ email: daless@iastate.edu
}

\begin{abstract}
In this paper, we define four different notions of controllability of physical interest for multilevel quantum mechanical systems. These notions involve the possibility of driving the evolution operator as well as the state of the system. We establish the connections among these different notions as well as methods to verify controllability.

The paper also contains results on the relation between the controllability in arbitrary small time of a system varying on a compact transformation Lie group and the corresponding system on the associated homogeneous space. As an application, we prove that, for the system of two interacting spin $\frac{1}{2}$ particles, not every state transfer can be obtained in arbitrary small time.
\end{abstract}

\section{Introduction}

In this paper, we consider multilevel quantum system described by a model [8] [17]

$$
\left|\dot{\psi}>=\left(A+\sum_{i=1}^{m} B_{i} u_{i}(t)\right)\right| \psi>
$$


where $\mid \psi>$ 円 is the state vector varying on the complex sphere $S_{\mathbb{C}}^{n-1}$, defined as the set of $n$-ples of complex numbers $x_{j}+i y_{j}, j=1, \ldots, n$, with $\sum_{j=1}^{n} x_{j}^{2}+y_{j}^{2}=1$. The matrices $A$, $B_{1}, \ldots, B_{m}$ are in the Lie algebra of skew-Hermitian matrices of dimension $n, u(n)$. If $A$ and $B_{i}, i=1, \ldots, m$ have zero trace they are in the Lie algebra of skew Hermitian matrices with zero trace, $s u(n)$. The functions $u_{i}(t), i=1,2, \ldots, m$ are the controls. They are assumed to be piecewise continuous and unconstrained in magnitude, although this assumption is immaterial for most of the theory developed here.

The solution of (1) at time $t, \mid \psi(t)>$ with initial condition $\left|\psi_{0}\right\rangle$, is given by:

$$
|\psi(t)>=X(t)| \psi(0)>
$$

where $X(t)$ is the solution at time $t$ of the equation

$$
\dot{X}(t)=\left(A+\sum_{i=1}^{m} B_{i} u_{i}(t)\right) X(t),
$$

with initial condition $X(0)=I_{n \times n}$.

The matrix $X(t)$ varies on the Lie group of special unitary matrices $S U(n)$ or the Lie group of unitary matrices $U(n)$ according to whether or not the matrices $A$ and $B_{i}$ in (3) have zero trace.

The controllability of the system (1) is usually investigated by applying general results on bilinear right invariant systems on compact Lie groups [14] [17]. These results, applied to our model, give a necessary and sufficient condition for the set of states reachable for system (3) to be the whole Lie group $U(n)$ (or $S U(n)$ ). The condition is given in terms of the Lie algebra generated by the matrices $A, B_{1}, \ldots, B_{m}$. Since both the groups $U(n)$ and $S U(n)$ are transitive on the complex sphere, it follows from (2) that this condition is also a sufficient condition for the controllability of the state $|\psi\rangle$.

In this paper, we first define four different notions of controllability which are of physical interest for quantum mechanical systems of the form (1). Using general results on transitivity of transformation groups, we provide criteria to check these controllability notions and we establish the connections among them. This is done in Sections 2 through 7 . Then, we investigate the relation between various notions of controllability in arbitrary small time and prove a general result in Section 8, which relates controllability in arbitrary time for a system varying on a compact transformation Lie group and the corresponding system on the associated homogeneous space. Although this is motivated by the analysis of multilevel quantum systems (where the transformation group is $S U(n)$ and the homogeneous space is the sphere $S_{\mathbb{C}}^{n-1}$ ) the analysis presented in Section 8 is valid for any compact transformation Lie group. As an application, we consider, in Section 9, the important model of two interacting spin $\frac{1}{2}$ particles in an electro-magnetic field. This system is of interest because it is used to perform two quantum bits logic operations in quantum computation [9]. An

\footnotetext{
${ }^{1}$ In this paper, we use Dirac notation $\mid \psi>$ to denote a vector on $\mathbb{C}^{n}$ of length 1 , and $<\psi|:=| \psi>>^{*}$ where ${ }^{*}$ denotes transposed conjugate
} 
analysis of the controllability of this system reveals what logic operation (state transfers) can be obtained. As an application of the previous analysis, we prove in this paper, the negative result that not every state transfer can be obtained for this model in arbitrary small time. Conclusions are given in Section 10.

\section{Definitions of Notions of Controllability for Multi- level Quantum Systems}

The following notions of controllability are of physical interest for quantum mechanical systems described in (11):

- Operator-Controllability (OC). The system is operator-controllable if every desired admissible (logic) operation on an arbitrary state can be performed using an appropriate control field. From (2) and (3), this means that there exists an admissible control to drive the state $X$ in (3) from the Identity to $X_{f}$, for any $X_{f} \in U(n)($ or $S U(n))$.

- State-Controllability (SC) The system is state-controllable if for every pair of initial and final state, $\mid \psi_{0}>$ and $\mid \psi_{1}>$ in $S_{\mathbb{C}}^{n-1}$ there exist control functions $u_{1}, \ldots, u_{m}$ and a time $t>0$ such that the solution of (11) at time $t$, with initial condition $\left|\psi_{0}\right\rangle$, is $|\psi(t)>=| \psi_{1}>$.

- Equivalent-State-Controllability (ESC) The system is equivalent-state-controllable if, for every pair of initial and final state, $\mid \psi_{0}>$ and $\mid \psi_{1}>$ in $S_{\mathbb{C}}^{n-1}$ there exist controls $u_{1}, \ldots, u_{m}$ and a phase factor $\phi$ such that the solution of (11) $\mid \psi>$, with $\mid \psi(0)>=$ $\mid \psi_{0}>$, satisfies $\left|\psi(t)>=e^{i \phi}\right| \psi_{1}>$, at some $t>0$.

A density matrix $\rho$ is a matrix of the form $\rho:=\sum_{j=1}^{r} w_{j}\left|\psi_{j}><\psi_{j}\right|$, where the coefficients $w_{j}>0, j=1,2, \ldots, r$, satisfy $\sum_{j=1}^{r} w_{j}=1$ (see e.g. [19] Chp. 3). The state is described using the density matrix formalism when the system is an ensamble of a number of non interacting quantum systems. The constants $w_{j}, j=1, \ldots, r$, give the proportion of such systems in the state $\mid \psi_{j}>$.

- Density-Matrix-Controllability (DMC) The system is density matrix controllable if, for each pair of unitarily equivalent 2 density matrices $\rho_{1}$ and $\rho_{2}$, there exists a control $u_{1}, u_{2}, \ldots, u_{m}$ and a time $t>0$, such that the solution of (3) at time $t, X(t)$, satisfies

$$
X(t) \rho_{1} X^{*}(t)=\rho_{2}
$$

\footnotetext{
${ }^{2}$ Two matrices $A, B \in U(n)$ are said to be unitarily equivalent if there exists a matrix $C \in U(n)$ such that $C A C^{*}=B$
} 
Equivalent state controllability is of interest because, in quantum mechanics, states that differ by a phase factor are physically indistinguishable. Therefore, from a physics point of view, having $E S C$ is as good as having $S C$.

Density matrix controllability is of interest when a mixed ensemble of different states is considered. In this case, the state at every time is represented by a density matrix which evolves as $\rho(t)=X(t) \rho(0) X^{*}(t)$, where $X(t)$ is solution of (3) with initial condition equal to the identity. Since $X(t)$ is unitary, only density matrices that are unitarily equivalent to the initial one can be obtained through time evolution.

In the following five sections we study the previous four notions of controllability, give criteria to check them in practice, and discuss the relations among them.

\section{Operator Controllability}

Operator controllability is the type of controllability considered in [17] (see also [1], [10], 20] for explicit controllability criteria). Operator controllability can be checked by verifying the Lie algebra rank condition [14], namely by verifying whether or not the Lie algebra generated by $\left\{A, B_{1}, B_{2}, \ldots, B_{m}\right\}$ is the whole Lie algebra $u(n)$ (or $s u(n)$ ). More in general, recall that there exists a one to one correspondence between the Lie subalgebras of $u(n)$ and the connected Lie subgroups of $U(n)$. We will denote in the sequel by $\mathcal{L}$ the Lie algebra generated by $\left\{A, B_{1}, B_{2}, \ldots, B_{m}\right\}$ and by $e^{\mathcal{L}}$ the corresponding connected Lie subgroup of $U(n)$. We have the following result, which, in essence, follows from the fact that $U(n)$ is a compact Lie group.

Theorem 1 The set of states attainable from the Identity for system (3) is given by the connected Lie subgroup $e^{\mathcal{L}}$, corresponding to the Lie algebra $\mathcal{L}$, generated by $\left\{A, B_{1}, B_{2}, \ldots, B_{m}\right\}$.

A proof is given in [2]

From Theorem [1, it is clear that the Lie algebra rank condition is also necessary to have operator controllability, thus we have:

Corollary 3.1 System ([) is operator-controllable if and only if $\mathcal{L}=u(n)($ or $\mathcal{L}=s u(n))$.

\section{State Controllability}

From the representation of the solution of Schrödinger equation (1) in (2), it is clear that the system is state controllable if and only if the Lie group $e^{\mathcal{L}}$ corresponding to the Lie algebra $\mathcal{L}$ generated by $\left\{A, B_{1}, \ldots, B_{m}\right\}$ is transitive on the complex sphere $S_{\mathbb{C}}^{n-1}$. Results on the classification of the compact and effective 0 Lie groups transitive on the (real) sphere were obtained in [3] [15] 18. Applications to control systems were described in [4]. We will recall

\footnotetext{
${ }^{3}$ Recall (see e.g. [16] pg. 40) that a transformation group $G$ on a manifold $M$ is called effective if the only transformation in $G$ that leaves every element of $M$ fixed is the identity in $G$.
} 
in Theorem 3 these results and then will provide further results and make the necessary connections for the application of interest here.

We consider the canonical Lie group isomorphism between $U(n)$ and a Lie subgroup of $S O(2 n)$. The correspondence between the matrices $X=R+i Y$ in $U(n)$, with $R$ and $Y$ real, and the matrix $\tilde{X} \in S O(2 n)$ is given by

$$
\tilde{X}:=\left(\begin{array}{cc}
R & -Y \\
Y & R
\end{array}\right)
$$

The same formula (5) provides the corresponding isomorphism between the Lie algebra $u(n)$ and a Lie subalgebra of $s o(2 n)$. As $X$ acts on $\mid \psi>:=\psi_{R}+i \psi_{I}$ on the complex sphere $S_{\mathbb{C}}^{n-1}, \tilde{X}$ acts on the vector $\left(\begin{array}{c}\psi_{R} \\ \psi_{I}\end{array}\right)$ on the real sphere $S^{2 n-1}$. Therefore, transitivity of one action is equivalent to transitivity of the other. Since $S O(2 n)$ is effective on the real sphere $S^{2 n-1}$ so is each of its Lie subgroups and in particular the one obtained from $e^{\mathcal{L}}$ via the transformation (5). As for compactness, notice that the transformation (5) preserves compactness. Moreover, $e^{\mathcal{L}}$ is connected and we have the following facts (see [16] pg. 226, we state here this result in a form suitable to our purposes):

Theorem 2 [10] For every connected Lie group $G$ which is transitive on the real sphere, there exists a compact connected Lie subgroup $H \subseteq G$ which is also transitive. f

Theorem 3 (15], 18]) The only compact connected Lie subgroups of $S O(2 n)$ that are transitive on the real sphere of odd dimensions $S^{2 n-1}$ are locally isomorphic to one of the following:

1) $S O(2 n)$ itself.

2) $U(n)$.

3) $S U(n), n \geq 2$.

4) The symplectic group $S p\left(\frac{n}{2}\right)$, for $n$ even and $n>2$. P

5) The full quaternion-unitary group defined as the group generated by $S p\left(\frac{n}{2}\right)$ and the one dimensional group $\left\{K \in U(n) \mid K:=e^{i \phi} I_{n}, \phi \in \mathbf{R}\right\}, n>2$ and even.

6) The covering groups of $S O(7)$ and $S O(9)$ for $n=4$ and $n=8$, respectively.

\footnotetext{
${ }^{4}$ Connectedness is not explicitly mentioned in the result in 16] but it follows from the proof since $H$ is in fact a maximal compact subgroup of $G$ which is always connected (see [16] pg. 188).

${ }^{5}$ Recall the Lie group of symplectic matrices $S p(k)$ is the Lie group of matrices $X$ in $S U(2 k)$ satisfying $X J X^{T}=J$, with $J$ given by $J=\left(\begin{array}{cc}0 & I_{k} \\ -I_{k} & 0\end{array}\right)$.
} 
Notice that Theorem 3 solves only partially the problem of determining which subgroups of $S O(2 n)$ are transitive on the real sphere $S^{2 n-1}$. In fact it only gives a necessary condition for the Lie algebra to be isomorphic to one of the Lie algebras of the Lie groups listed in the theorem. It is known that, for example, the realification (5) of the symplectic group $S p\left(\frac{n}{2}\right)$ is transitive on $S^{2 n-1}$, but nothing can be said from the Theorem for Lie groups that are only locally isomorphic (namely have isomorphic Lie algebra) to $S p\left(\frac{n}{2}\right)$, unless further information is supplied. In this paper we are interested only in the subgroups of $S O(2 n)$ that are isomorphic via (5) to a subgroup of $S U(n)$ (or $U(n)$ ). We will solve the problem of giving necessary and sufficient conditions for state controllability in terms of the Lie algebra $\mathcal{L}$ generated by $A, B_{1}, B_{2}, \ldots, B_{m}$ in Theorem 4 . In the following three Lemmas we use representation theory and structure theory (see e.g. [13]) to prove three properties of classical Lie groups and algebras which we will use in the proof of Theorem 4 . We refer to [13] for the terminology and notions of Lie group theory used here.

Recalling that, by definition, the covering groups of $S O(7)$ and $S O(9)$ have Lie algebras isomorphic to $s o(7)$ and $s o(9)$ respectively, the following lemma will be used to rule out that such groups arise, after realification (5), as subgroups of $S U(4)$ (or $U(4)$ ) and $S U(8)$ (or $U(8))$.

Lemma 4.1 (a) There is no Lie subalgebra of $s u(4)$ (or $u(4))$ isomorphic to $s o(7)$.

(b) There is no Lie subalgebra of $s u(8)$ ( or $u(8))$ isomorphic to $s o(9)$.

A proof is given in [2]

Lemma 4.2 Assume $n$ even. All the subalgebras of $s u(n)$ or $u(n)$ that are isomorphic to $s p\left(\frac{n}{2}\right)$ are conjugate to $s p\left(\frac{n}{2}\right)$ via an element of $U(n)$.

A proof is given in [2]

Lemma 4.3 Assume $n$ even. Then, the only subalgebra of $s u(n)$ containing $s p\left(\frac{n}{2}\right)$ properly is $s u(n)$ itself.

A proof is given in [2]

Notice that the above property also holds for any Lie algebra which is isomorphic to $s p\left(\frac{n}{2}\right)$, since from Lemma (4.2) any such subalgebra is in fact conjugate to $s p\left(\frac{n}{2}\right)$.

We are now ready to state a necessary and sufficient condition of state controllability in terms of the Lie algebra $\mathcal{L}$ generated by $\left\{A, B_{1}, B_{2}, \ldots, B_{m}\right\}$.

Theorem 4 The system is state controllable if and only if $\mathcal{L}$ is isomorphic (conjugate) to $\operatorname{sp}\left(\frac{n}{2}\right)$ or to $s u(n)$, for $n$ even, or to $s u(n)$, for $n$ odd (with or without the $i I$, where $I$ is the identity matrix). 
Proof. If the system is state controllable then $e^{\mathcal{L}}$ is transitive on the complex sphere $S_{\mathbb{C}}^{n-1}$, therefore its realification (5) is transitive on the real sphere $S^{2 n-1}$. Thus, from Theorem 2, it must contain a Lie group locally isomorphic to one of the groups listed in Theorem 3 . As a consequence, the Lie algebra $\mathcal{L}$ must contain a Lie algebra isomorphic to one of the corresponding Lie algebras. Assume first $n$ odd, then cases 4) 5) and 6) are excluded. Case 1 ) is also excluded since $\operatorname{dim} S O(2 n)>\operatorname{dim} U(n)$, when $n \geq 2$ (recall that $S O(2)$ is the realification of $U(1))$. Therefore $\mathcal{L}$ must be either $s u(n)$ or $u(n)$ in this case. If $n=2$ then $s u(2)=s p(1)$ so cases 3) and 4) and 2) and 5) coincide. If $n$ is even and $n>2$, then case 1) is excluded as above and cases 2) through 5) all imply that $s p\left(\frac{n}{2}\right) \subseteq \mathcal{L}$ up to isomorphism of $\operatorname{sp}\left(\frac{n}{2}\right)$, which from Lemma 4.3 gives $\mathcal{L}=\operatorname{sp}\left(\frac{n}{2}\right)$ or $\mathcal{L}=s u(n)$ up to isomorphism (with or without the identity matrix). Case 6) is excluded by Lemma 4.1. This proves that the only possible Lie algebras $\mathcal{L}$ that correspond to a transitive Lie group are the ones given in the statement of the Theorem. The converse follows from the well known properties of transitivity of $S U(n)$ and $S p\left(\frac{n}{2}\right)$ as well as of any group conjugate to them via elements in $U(n)$, and from Lemma 4.2 .

\section{Equivalent State Controllability}

The notion of equivalent state controllability, although seemingly weaker, is in fact equivalent to state controllability. In order to see this, notice that if the system is $E S C$ then for every pair of states $\mid \psi_{0}>$ and $\mid \psi_{1}>$ there exists a matrix $X$ in $e^{\mathcal{L}}$ and a 'phase' $\phi \in \mathbf{R}$ such that

$$
X\left|\psi_{0}>=e^{i \phi}\right| \psi_{1}>
$$

This can be expressed by saying that there exists an element $Y$ in $e^{i \phi} e^{\mathcal{L}}:=\{Y \in U(n) \mid Y=$ $\left.e^{i \phi} X, X \in e^{\mathcal{L}}, \phi \in \mathbf{R}\right\}$ such that $Y\left|\psi_{0}>=\right| \psi_{1}>$ and therefore $e^{i \phi} e^{\mathcal{L}}$ is transitive on the complex sphere. Now, if $\operatorname{span}\left\{i I_{n}\right\} \subseteq \mathcal{L}$, then $e^{i \phi} e^{\mathcal{L}}=e^{\mathcal{L}}$ and therefore $e^{\mathcal{L}}$ is transitive and the system is $S C$. If this is not the case, then from Theorem 2, there must exist a compact connected Lie group $G \subseteq e^{\mathcal{L}}$ such that $e^{i \phi} G$ is transitive. From Theorem I' in [15], it follows, writing $e^{i \phi} G$ as $e^{i \phi} I_{n} \times G$, that one between the two groups $e^{i \phi} I_{n}$ and $G$, must be transitive. Therefore $G \subseteq e^{\mathcal{L}}$ is transitive. In conclusion, we have the following Theorem.

Theorem 5 ESC and SC are equivalent properties for quantum mechanical systems (1).

Theorems 1 and 5 show that a necessary and sufficient condition to have state controllability or equivalent state controllability is that the Lie algebra $\mathcal{L}$ is the whole $s u(n)$ or isomorphic to $\operatorname{sp}\left(\frac{n}{2}\right)$ (with or without $i I$ ). To check this isomorphism one can apply the structure theory of Lie algebras to $\mathcal{L}$. A more practical way to check equivalent state controllability will be presented in Section 7. This method only involves elementary matrix manipulations and can be extended to check density matrix controllability starting from a fixed given matrix. 


\section{Density Matrix Controllability}

Notice that if $e^{\mathcal{L}}=S U(n)$ or $e^{\mathcal{L}}=U(n)$ then obviously the system is $D M C$. Moreover, in order for the system to be $D M C$, the model has to be equivalent state controllable (and therefore state controllable) as well, because transitions between pure states represented by matrices of the form $|\psi><\psi|$ must be possible. Therefore, to get $D M C, \mathcal{L}$ must be $s u(n)$, or, for $n$ even and $n>2$ (see Theorem $\mathbb{4}$ ), it must be isomorphic (conjugate) to $\operatorname{sp}\left(\frac{n}{2}\right)$. The next example shows that $S p\left(\frac{n}{2}\right)$ is not enough to obtain $D M C$. The example constructs a class of density matrices $D$ with the property that

$$
\left\{W D W^{*} \mid W \in S p\left(\frac{n}{2}\right)\right\} \neq\left\{U D U^{*} \mid U \in S U(n)\right\}
$$

Example 6.1 Choose any $n>2$ with $n$ even, and let $\mid v>=\left(\begin{array}{l}v_{1} \\ v_{2}\end{array}\right) \in \mathbb{C}^{n}$ and $\mid w>=$ $\left(\begin{array}{c}-v_{2} \\ v_{1}\end{array}\right) \in \mathbb{C}^{n}$, with $v_{1}, v_{2} \in \mathbf{R}^{n / 2},\|v\|=1$. Then $\|w\|=1,<v \mid w>=0$, thus, in particular, these two vectors are independent. Let

$$
D=\frac{1}{2}(|v><v|+|w><w|) .
$$

It is easy to verify that $D J=J D$ (where $J=\left(\begin{array}{cc}0 & I_{n} \\ -I_{n} & 0\end{array}\right)$ ). Thus, if $W \in S p\left(\frac{n}{2}\right)$ then we still have that:

$$
\left(W D W^{*}\right) J=J \overline{\left(W D W^{*}\right)} .
$$

Choose any two orthonormal vectors $\left|v^{\prime}>,\right| w^{\prime}>\in \mathbb{C}^{n}$, such that:

$$
D^{\prime}=\frac{1}{2}\left(\left|v^{\prime}><v^{\prime}\right|+\left|w^{\prime}><w^{\prime}\right|\right)
$$

satisfies $D^{\prime} J \neq J \bar{D}^{\prime}$ (it is easy to see that two such vectors exist), and let $U \in U(n)$ be any unitary matrix such that $U v=v^{\prime}$ and $U w=w^{\prime}$, then

$$
U D U^{*}=D^{\prime} \neq W D W^{*}
$$

for all $W \in S p\left(\frac{n}{2}\right)$.

From the above discussion and example, we can conclude that $D M C$ is equivalent to $O C$.

Given a density matrix $D$, it is of interest to give a criterion on the Lie algebra $\mathcal{L}$ for the two orbits

$$
\mathcal{O}_{\mathcal{L}}:=\left\{W D W^{*} \mid W \in e^{\mathcal{L}}\right\}
$$

and

$$
\mathcal{O}_{U}:=\left\{U D U^{*} \mid U \in U(n)\right\}
$$


to coincide. To this aim, notice that since $D$ is Hermitian, $i D$ is skew-Hermitian so that $i D \in u(n)$, and a matrix commutes with $i D$ if and only if it commutes with $D$. The centralizer of $i D$ is by definition, the Lie subalgebra of $u(n)$ of matrices that commute with $i D$. Call this subalgebra $\mathcal{C}_{D}$ and the corresponding connected Lie subgroup of $U(n), e^{\mathcal{C}_{D}}$. Analogously, the centralizer of $i D$ in $\mathcal{L}$ is $\mathcal{C}_{D} \cap \mathcal{L}$ and we denote by $e^{\mathcal{C}_{D} \cap \mathcal{L}}$ the corresponding subgroup of $U(n)$ (which is also a subgroup of $e^{\mathcal{L}}$ ).

For a given density matrix $D$, it is sufficient to calculate the dimensions of $\mathcal{L}, \mathcal{C}_{D}$ and $\mathcal{C}_{D} \cap \mathcal{L}$ to verify the equality of the two orbits $\mathcal{O}_{\mathcal{L}}$ and $\mathcal{O}_{U}$ defined in (8) (9). We have the following result.

Theorem 6 Let $D$ be a given density matrix, then $\mathcal{O}_{\mathcal{L}}=\mathcal{O}_{U}$ if and only if

$$
\operatorname{dim} u(n)-\operatorname{dim} \mathcal{C}_{D}=\operatorname{dim} \mathcal{L}-\operatorname{dim}\left(\mathcal{L} \cap \mathcal{C}_{D}\right)
$$

Proof. We have the following isomorphisms between the two coset spaces $U(n) / e^{\mathcal{C}_{D}}$ and $e^{\mathcal{L}} / e^{\mathcal{C}_{D} \cap \mathcal{L}}$ and the two manifolds $\mathcal{O}_{U}$ and $\mathcal{O}_{\mathcal{L}}$, respectively. So, it holds

$$
\begin{aligned}
& U(n) / e^{\mathcal{C}_{D}} \simeq\left\{U D U^{*} \mid U \in U(n)\right\}, \\
& e^{\mathcal{L}} / e^{\mathcal{C}_{D} \cap \mathcal{L}} \simeq\left\{W D W^{*} \mid W \in e^{\mathcal{L}}\right\},
\end{aligned}
$$

where $\simeq$ means isomorphic. Therefore if the two orbits coincide, we must have that the two coset spaces must coincide as well. So, in particular, their dimensions have to be equal which gives $(10)$.

Conversely assume that (10) is verified. Then the dimensions of the two coset spaces on the left hand sides of (11) and (12) are the same and so are the dimensions of the manifolds on the right hand side namely $\mathcal{O}_{U}$ and $\mathcal{O}_{\mathcal{L}}$. Notice also that these two manifolds are connected since both $U(n)$ and $e^{\mathcal{L}}$ are connected. Since $e^{\mathcal{C}_{D}}$ is closed in $U(n)$ and therefore compact, from Proposition 4.4 (b) in [11] we have that $e^{\mathcal{L}} / e^{\mathcal{L} \mathcal{C}_{D}}$ is closed in $U(n) / e^{\mathcal{C}_{D}}$. On the other hand, since the two coset spaces have the same dimensions, $e^{\mathcal{L}} / e^{\mathcal{L} \mathcal{C}_{D}}$ is open in $U(n) / e^{\mathcal{C}_{D}}$. By connectedness, we deduce that the two coset spaces must coincide, and therefore the two orbits coincide as well.

Special cases of the above Theorem, are density matrices representing pure states or completely random states. In the first case, the density matrix $D$ as the form, $D=|\psi><\psi|$ and, in an appropriate basis, it can be written as a diagonal matrix with the $(1,1)$ entry equal to one and all the remaining entries equal to zero. The analysis in Section 4 shows that the only Lie algebras $\mathcal{L}$ satisfying condition (10) are $s u(n)$ with or without $i I$ or, for $n$ even, isomorphic to $\operatorname{sp}\left(\frac{n}{2}\right)$. For completely random states, the density matrix $D$ is a real scalar matrix with trace equal to one, and therefore its centralizer in $\mathcal{L}, \mathcal{L} \cap \mathcal{C}_{D}$, is all of $\mathcal{L}$, for every subalgebra $\mathcal{L}$. Thus the condition $(10)$ holds with $\operatorname{dim} \mathcal{L}-\operatorname{dim} \mathcal{L} \cap \mathcal{C}_{D}=0$ for every $\mathcal{L}$. The interpretation, from a physics point of view, is the obvious fact that a completely random ensemble of quantum systems remains completely random after any evolution. 


\section{Test of Controllability}

As we have shown in the previous sections, the two notions of operator-controllability and density-matrix-controllability are equivalent and they are the strongest among the controllability notions we have defined. On the other hand, state-controllability and equivalentstate-controllability are equivalent. These facts are summarized in the following diagram:

$$
\mathrm{DMC} \quad \Leftrightarrow \quad \mathrm{OC} \quad \Rightarrow \quad \mathrm{SC} \quad \Leftrightarrow \quad \mathrm{ESC}
$$

From a practical point of view, it is of great interest to give criteria on the Lie algebra $\mathcal{L}$ to ensure that the corresponding group is transitive on the complex sphere. In this case the system is state controllable. As we have seen from the analysis in Section 4, the Lie algebra $\mathcal{L}$ has to be to $s u(n)$ or $u(n)$ or, for $n$ even, conjugate and therefore isomorphic to $\operatorname{sp}\left(\frac{n}{2}\right)$. To check this isomorphism, one can apply the Cartan theory of classification of semisimple Lie algebras [1]]. A simpler test can be derived from Theorem [6. To this purpose, notice that state controllability is the same as equivalent state controllability (see Theorem 5) and this can be easily seen to be equivalent to the possibility of steering the matrix

$$
D=\operatorname{diag}(1,0,0, \ldots, 0)
$$

to any unitarily equivalent matrix. The centralizer $\mathcal{C}_{D}$ of the matrix $i D$ in (13) in $u(n)$, is given by the set of matrices of the form

$$
M:=\left(\begin{array}{cc}
i a & 0 \\
0 & H
\end{array}\right)
$$

with $a$ any real and $H$ a matrix in $u(n-1)$. The dimension of $\mathcal{C}_{D}$ is $(n-1)^{2}+1$ and therefore the number on the right hand side of (10) is $n^{2}-\left((n-1)^{2}+1\right)=2 n-2$. In conclusion as a consequence of Theorems 6 and 5 we have the following easily verifiable criterion for state controllability.

Theorem 7 With the above notations and definitions, the system (3) is state controllable if and only if the Lie algebra $\mathcal{L}$ generated by $\left\{A, B_{1}, B_{2}, \ldots, B_{m}\right\}$ satisfies

$$
\operatorname{dim} \mathcal{L}-\operatorname{dim}\left(\mathcal{L} \cap \mathcal{C}_{D}\right)=2 n-2
$$

We remark here that similar criteria can be given for different density matrices according to Theorem 6 .

Example 7.1 Assume that the Lie algebra $\mathcal{L}$ is given by the matrices of the form

$$
F:=\left(\begin{array}{cc}
L+Z & T+C \\
-\bar{T}+\bar{C} & -L+Z^{T}
\end{array}\right)
$$

with $L$ diagonal and purely imaginary, $T$ diagonal, and $Z, C$ having zeros on the main diagonal, all of them $2 \times 2$ matrices. This Lie algebra is in fact conjugate to $s p(2)$. Verifying 
this fact directly can be cumbersome. However to prove that the associated system is state controllable, one can verify that the Lie subalgebra of matrices of $\mathcal{L}$ that have the form (14), namely $\mathcal{L} \cap \mathcal{C}_{D}$, has dimension 4 . Since the dimension of $\mathcal{L}$ is 10 , we have (recall $n=4$ )

$$
\operatorname{dim} \mathcal{L}-\operatorname{dim} \mathcal{L} \cap \mathcal{C}_{D}=6=2 n-2 .
$$

Therefore the criterion of Theorem 7 is verified.

\section{$8 \quad$ State transfer in arbitrary time}

In this section, we study the possibility of transferring the state of (11) and (3) in arbitrary small time. For the results that will follow, we can assume that $X$ varies on a general compact transformation (matrix) Lie group $G$ (with corresponding subalgebra $\mathcal{G}$ ) while $|\psi\rangle$ varies on the corresponding homogeneous space $M$. First, we define the set of states reachable in arbitrary time for system (3). We denote it by $\mathcal{A} \subseteq G$; we have:

$$
\mathcal{A}=\cap_{t>0} R(t)
$$

where $R(t)$ is the set of states reachable from the identity at time $t$ for system (3).

As it has been shown with a number of examples [5] [14], even though the set of states reachable from the identity for (3) is the whole group $G$ and the magnitude of the controls is unconstrained, it is possible that not all the states in $G$ can be obtained in arbitrary small time. In fact conditions can be given on $A, B_{1}, \ldots, B_{m}$ for $\mathcal{A}$ to be empty [6]. However, it may well be that even if $\mathcal{A}$ is a proper subset of $G, \mathcal{A}$ is still transitive on $M$. This fact is easily seen to be necessary and sufficient for state transfer in arbitrary small time between two states in $M$.

In this section, we investigate the relation between these two notions of controllability in arbitrary time. A general study of the controllability in arbitrary time of systems on compact Lie groups was presented in [6].

Instead of working with $\mathcal{A}$, it is more convenient to work with its 'regularized version'

$$
\mathcal{A}_{\text {reg }}:=\bigcap_{t>0} \bar{R}(t)
$$

where $\bar{R}(t)$ is the closure of $R(t)$. The set $\mathcal{A}_{\text {reg }}$ has more structure because it is a compact connected Lie subgroup of $G$ 田

Theorem 8 If $\mathcal{A}$ is not empty, $\mathcal{A}_{\text {reg }}$ is a compact connected Lie subgroup of $G$.

A proof is presented in [2]

If $\mathcal{A}$ is not empty, then $\mathcal{A}_{\text {reg }}$ is a connected Lie group and we can consider its associated Lie algebra $\mathcal{L}_{A}$. Consider $\mathcal{B}$ the Lie algebra generated by $B_{1}, \ldots, B_{m}$ in (3). We have.

\footnotetext{
${ }^{6}$ It is proved in $[6]$ that Small Time Local Controllability of the identity of the group $G$ implies $\mathcal{A}=\mathcal{A}_{\text {reg }}$.
} 
Theorem $9 \mathcal{B} \subseteq \mathcal{L}_{A}$

Proof. It follows immediately from the fact (see [6]) that the connected subgroup corresponding to $\mathcal{B}$ is a subgroup of $\mathcal{A}_{\text {reg }}$.

In the following theorem we will call transitive a subalgebra of $\mathcal{G}$ whose corresponding Lie subgroup of $G$ is transitive on $M$. We have

Theorem 10 Assume $\mathcal{B}$ is not a subalgebra of any transitive proper subalgebra of $\mathcal{G}$. If the system is state controllable in arbitrary time, then $\mathcal{A}_{\text {reg }}=G$, in particular for every $t$ $\bar{R}(t)=G$.

Proof. Assume that the system is state controllable in arbitrary time. This means that the set $\mathcal{A}$ is transitive on $M$ and since $\mathcal{A} \subseteq \mathcal{A}_{\text {reg }}$, so is $\mathcal{A}_{\text {reg }}$. It follows from the assumptions on $\mathcal{B}$ and the fact that $\mathcal{B} \subseteq \mathcal{L}_{A}$ that $\mathcal{A}_{\text {reg }}$ has to be equal to $G$.

\section{Application to systems of two spin $\frac{1}{2}$ particles}

In [7], and [12] the system of two spin $\frac{1}{2}$ interacting particles in a driving electro-magnetic field was considered. The system has the form (11), with $m=3$, where the matrices $A, B_{1}, B_{2}$, $B_{3}$, are appropriate matrices in $s u(4)$ and the solution $\mid \psi>$ varies on the sphere $S^{3} \mathbb{C}$ while $X$ in (3) varies in $S U(4)$. The matrix $A$ models the interaction between the two particles which can assume different forms (e.g. isotropic, dipolar) while $B_{1}, B_{2}$ and $B_{3}$ model the interaction between particles and the external field. The controls $u_{1}, u_{2}, u_{3}$ are component of a driving electro-magnetic field in the $x, y$ and $z$ direction, respectively. In typical experimental set ups, the $z$-component of the field is held constant. This system is of interest because it is used to perform two quantum bit logic operations in quantum computing [9]. Also, it is the simplest case, for the study of controllability in NMR experiments, after the case of a single spin $\frac{1}{2}$ particle dealt with in [5]. A study of the Lie algebra structure for general systems of any number of spin $\frac{1}{2}$ particles and of its controllability properties is presented in [1].

All the models of two interacting spin $\frac{1}{2}$ particles have in common the following property. There exists a Cartan decomposition of $s u(4)$ (see [11) of the form

$$
s u(4)=\mathcal{P} \oplus \mathcal{B},
$$

such that $\mathcal{B}$ is generated by the matrices $B_{i}(i=1,2,3)$, and $A \in \mathcal{P}$. For these systems it follows from [12], Theorem 10, that, for any $U \in S U(4)$, if $T_{U}=\inf \{t \geq 0 \mid U \in R(t)\}$, then

$$
U=Q_{1} \exp \left\{T_{U} Y\right\} Q_{2},
$$

where $Q_{1}, Q_{2} \in e^{\mathcal{B}}$ and $Y \in \mathcal{P}$.

It follows from (20) that these models are not operator controllable in arbitrary small time. In fact, if this was the case, $T_{U}$ would have to be zero for any $U \in S U(4)$, but this fact would imply $U \in e^{\mathcal{B}} \forall U$, which is false. As an application of the previous results, we 
show here that these systems are not state controllable nor equivalent state controllable in arbitrary time, as well.

It is possible to prove that $\mathcal{B}$ in this case satisfies the property in Theorem 10 , since there is no subalgebra containing $\mathcal{B}$ other than $s u(4)$ itself and $\mathcal{B}$ is conjugate to $s o(4) \neq s p(2)$, so that it follows from Theorem $\mathbb{1}$ that $\mathcal{B}$ is not a transitive subalgebra. Therefore, the system satisfies the conditions of Theorem 10, and it follows that if the system is state controllable in arbitrary time then $\mathcal{A}_{r e g}=S U(4)$. In other terms, for every time $t, \bar{R}(t)=S U(4)$. On the other hand

$$
\bar{R}(t)=S U(4) \Rightarrow T_{U}=0 \text { for all } U
$$

which is false, as seen before. To prove (21), we argue by contradiction. Assume that, for a given $U \in S U(4), T_{U}>0$. Since $S U(4)=\operatorname{int}\left(R^{-1}\left(T_{U} / 2\right) U\right)$ and $S U(4)=\bar{R}\left(T_{U} / 4\right)$, there exists an open set $N$ such that:

$$
N \subseteq \operatorname{int}\left(R^{-1}\left(T_{U} / 2\right) U\right) \cap R\left(T_{U} / 4\right)
$$

Choose any matrix $\tilde{U} \in N$, then from $I$ we reach $\tilde{U}$ in time $T_{U} / 4$, and from $\tilde{U}$ we reach $U$ in time $T_{U} / 2$, thus $U \in R\left(\frac{3 T_{U}}{4}\right)$, which contradicts the minimality of $T_{U}$.

\section{Conclusions}

For quantum mechanical systems in the multilevel approximation a number of concepts concerning controllability can be considered. One can ask whether it is possible to drive the evolution operator or the state to any desired configuration. One typically represents the state with a vector with norm 1 or using the density matrix formalism. Connections between different notions of controllability have been established in this paper, where we have shown that the possibility of driving a pure state between two arbitrary configurations is in general a weaker property than the controllability of the evolution operator. All the controllability properties of a given quantum system can studied by studying the Lie algebra generated by the matrices $\left\{A, B_{1}, \ldots, B_{m}\right\}$ of the system (1). This Lie algebra has to be the full Lie algebra $s u(n)$ (or $u(n))$ for controllability of the operator while for controllability of the state it can be conjugate and therefore isomorphic to the Lie algebra of symplectic matrices of dimension $n$ modulo a phase factor. We have also given a practical test to check this isomorphism. This test can be extended for density matrices of rank different from one and only requires elementary algebraic manipulations involving the centralizer of the given density matrix.

The paper also contains some results on the relation between controllability in arbitrary small time for a system on a Lie transformation group and for the corresponding system on the associated homogeneous space. The application of these results to the systems of two

interacting spin $\frac{1}{2}$ particles in an electro-magnetic field shows the negative result that it is not possible for this system to obtain a state transfer between two points even though the control can be taken with arbitrary large magnitude. 


\section{References}

[1] F.Albertini and D. D'Alessandro, The Lie algebra structure and nonlinear controllability of spin systems, Technical Report Department of Mathematics Iowa State University, 2001, xxx.lanl.gov guant-ph 0106115.

[2] F. Albertini and D. D'Alessandro, Notions of controllability for quantum mechanical systems, Technical Report Department of Mathematics Iowa State University, 2001, submitted for publication.

[3] A. Borel, Some remarks about transformation groups transitive on spheres and tori, Bull. Amer. Math. Soc. 55, pp. 580-586, 1949.

[4] R. Brockett, Lie theory and control systems defined on spheres, SIAM J. Appl. Math. Vol. 25. No. 2, September 1973.

[5] D. D'Alessandro, Topological properties of reachable sets and the control of quantum bits, Systems and Control Letters, 41, pp. 213-221, 2000.

[6] D. D'Alessandro, Small time controllability of systems on compact Lie groups and spin angular momentum, to appear in Journal of Mathematical Physics

[7] D. D'Alessandro, Constructive controllability of one and two spin $\frac{1}{2}$ particles, Proc. $A C C$ 2001, Arlington, Virginia, U.S.A..

[8] D. D'Alessandro and M. Dahleh, Optimal control of two-level quantum systems, in IEEE Transaction on Automatic Control, to appear.

[9] D. DiVincenzo, Quantum Computation, Science Vol. 270, 13 October, 1995.

[10] H. Fu, S. G. Schirmer and A. I. Solomon, Complete Controllability of finite level quantum systems, J. Phys. A 34, pp. 1679-1693, also xxx.lanl.gov quant-ph/0102017.

[11] S. Helgason, Differential Geometry, Lie Groups and Symmetric Spaces, Academic Press, New York, 1978.

[12] N. Khaneja, R. Brockett and S. J. Glaser, Time optimal control in spin systems, Physical Review A Vol. 63, 032308, 2001.

[13] A. Knapp, Representation Theory. An Overview Based on Examples, Princeton University Press, Princeton New Jersey, 1986.

[14] V. Jurdevic and H. Sussmann, Control systems on Lie groups, Journal of Differential Equations, 12, 313-329, 1972.

[15] D. Montgomery and H. Samelson, Transformation groups of spheres. Ann. of Math. 44, 1943, pp. 454-470. 
[16] D. Montgomery and L. Zippin, Topological Transformation Groups, Interscience Tracts in Pure and Applied Mathematics, Vol. 1, New york, N.Y. 1955.

[17] V. Ramakrishna, M. Salapaka, M. Dahleh, H. Rabitz and A. Peirce, Controllability of molecular systems, Physical Review A, Vol. 51, No. 2, February 1995.

[18] H. Samelson, Topology of Lie groups, Bull. Amer. Math. Soc., 58, 1952, pp. 2-37.

[19] J. J. Sakurai, Modern Quantum Mechanics, Addison-Wesley Pub. Co., Reading, Mass. 1994.

[20] S. G. Schirmer, H. Fu and A. I. Solomon, Complete controllability of quantum systems, Phys. Rev. A, 63, art. no. 063410 (2001). 